\title{
Adaptive human-like control system design of a lower limb robot using minimum inertial parameters
}

\begin{abstract}
In this paper, aiming at the comfort problem of wearing rehabilitation training robot for different patients with lower extremity dyskinesia, the real human gait data as the reference control input is proposed, and a human-like control method based on the minimum inertia parameter with the comfort of a human wearable robot as the objective function is designed. To realize the real-time tracking of the reference trajectory by the robot trajectory of lower limb rehabilitation training, the NOKOV motion capture system is used to collect human gait data. The Lagrange method is used to establish the dynamics model of the single leg of the lower limb rehabilitation training robot. Based on the minimum inertia parameters, the adaptive controller with comfort as the objective function is designed. Finally, the effectiveness of this method is verified by simulation based on experimental data.
\end{abstract}

Keywords: gait data analysis, comfortable function, minimum inertia, adapt tracking control
Volume 5 Issue 5 - 2019

\author{
Junfeng Zhang,' Xia Wu,' Aihui Wang,' \\ Xiaoyu Chen, ${ }^{2}$ Yan Wang' \\ 'Department of Electromechanical and Information Engineering \\ Henan Vocational College of Water Conservancy and \\ Environment, China \\ ${ }^{2}$ School of Electric and Information Engineering, Zhongyuan \\ University of Technology, China
}

Correspondence: Aihui Wang, School of Electric and Information Engineering, Zhongyuan University of Technology, Zhengzhou 450007, China, Email a.wan@zut.edu.cn

Received: October 24, 2019 | Published: October 31, 2019

\section{Introduction}

Medical studies have shown that appropriaterepetitive rehabilitation exercise training can help them realize the recombination of brain nerve function while patients receive drug therapy, so that patients can gradually recover the ability to walk independently, ${ }^{1-2}$ which not only improves the quality of life of patients but also greatly reduces family and social pressure. Traditional rehabilitation therapists mainly rely on clinical experience to conduct a gait analysis of patients. Although the method is simple, it can only do rough qualitative analysis. Due to lack the accurate, quantitative and controllable data support, it is difficult to ensure the scientificity and effectiveness of patients' rehabilitation training.

Lower limb rehabilitation robot has been a research hotspot in the world in recent years and is recognized as one of the better rehabilitation equipment. Whether the rehabilitation robot can achieve the goal of rehabilitation, the control strategy of the robot plays a crucial role, ${ }^{3}$ which is based on position control to achieve trajectory tracking. ${ }^{4}$ Therefore, the comfort of the lower limb rehabilitation training robot must be considered when designing the controller, which has certain social and scientific significance for the robot.

The geometrical parameters of the robot system are known or can be accurately measured, while many inertial parameters of the robot system are often imprecise or completely unknown. It is required to develop advanced controllers that can achieve the desired tracking accuracy of the robot even if the variations of robot inertia parameters. And the lower limb rehabilitation robot is a complex robot system with multi-input, multi-output, nonlinear, coupling and uncertain factors. The system needs to face different patients, each patient has different individual parameters and with external interference. Therefore, the control system should be robust and adaptive. At this point, the adaptive control scheme is a usually used approach, the system controller can adapt to the changes of the controlled object and external disturbance by modifying the parameters of the controller, so guarantee the robot tracking accuracy online real-time..$^{5-8}$ In this way, the lower limbs of patients are ensured to move smoothly and comfortably, speed mutation is avoided, and control accuracy is improved, so that patients can complete rehabilitation training safely and comfortably.

Many methods have been used to control the robot system. For example, the simplest control is designed by the Proportional Derivative (PD) control, ${ }^{9-10}$ although this method has been widely used in a robot control system, the performance of solving complex problems with uncertain parameters is not good. Yi G, et al ${ }^{11}$ proposed a trajectory tracking adaptive control method for lower limb rehabilitation robot with an uncertain model, however, it did not consider human comfort in human-computer interaction. Liu Y and Zhang $\mathrm{X}^{12}$ analyzed the mechanical structure design principle and requirements of flexible joints from the perspective of anthropomorphism. Yang F and Yuan $\mathrm{X}^{13}$ proposed a human motion control algorithm based on comfort maximization. Tang $\mathrm{A}$ and $\mathrm{Cao} \mathrm{Q}^{14}$ examined the requirements for comfortable walking and outlined the design of a motion control algorithm for a walking assistant robot based on comfort. The above research has not conducted in-depth research on the comfort level of human-computer interaction in the lower limb rehabilitation robot control system under minimum inertia parameters.

In most human gait acquisition devices, optical motion capture is preferred because it can accurately measure small reflective markers attached to some relevant body landmarks. Therefore, in this paper, the NOKOV $3 \mathrm{~d}$ infrared passive optical motion capture system is used to collect human movement gait data as input data of exoskeleton robot controller, and then proposes a human body comfort function based on human-computer interaction, in the case of uncertain model parameter, an adaptive controller is designed for lower limb rehabilitation robot trajectory tracking control to realize more comfortable natural gait trajectory and improve the efficiency 
of rehabilitation. Finally, the simulation experiment and lower limb exoskeleton rehabilitation training robot experiments verify the feasibility and effectiveness of the proposed method.

\section{System introduction and human walking data acquisition and pre processing}

NOKOV $3 \mathrm{~d}$ motion capture system is a passive optical motion capture system (Figure 1), which mainly consists of a host server, 6 optical cameras, reflective markers, T-shaped calibration bar, L-shaped calibration bar, controller and so on. The optical camera records the spatial coordinates of all the markers with a resolution of 2 million pixels and a capture frequency of $60 \mathrm{HZ}$, thereby obtaining a moving trajectory of the human body with the capture accuracy up to $1 \mathrm{~mm}$. The system is mainly decorated by using 3 points of six optical cameras around each of two columns to capture the 15 reflective markers on human body, and as long as the reflective marker can be seen by the two cameras at the same time, the specific position of the point in the space can be determined according to the images taken by the two cameras simultaneously and the camera's own parameters.In this paper, motion capture equipment is used to capture the trajectory of human joints. Since the capture system can only be attached to the outside of human joints, and optical motion capture equipment can only get the coordinates of the outside of human lower limb joints, it is necessary to calculate the central position of each joint of human lower limbs according to mathematical formulas. In this paper, the human body experience formula is used to calculate the central position of each joint. In the process of capture, the captured data needs to be filtered according to the error of the capture system itself, the stray point error in the space and the external factors such as the jitter in the process of human walking. The commonly used filtering methods mainly include least square filtering, wavelet filtering, Kalman filtering and so on. In this paper, the data from the hip joint, the knee joint and the ankle joint of the left leg are processed by using a least square filter and wavelet filter with better effect. After treatment, the Angle of the hip joint and knee joint was calculated, as shown in Figure 2.

In order to protect the lower limb rehabilitation robot, the joint angle cannot be directly applied to the lower limb rehabilitation robot, so curve fitting should be carried out to ensure a smooth joint angle curve. The common curve fitting forms are Fourier function, Gaussian function and Sum of Sine function. By comparing the fitting coefficients and root mean square error of the three, the gaussian function with a good fitting term of 8 was selected in this paper.

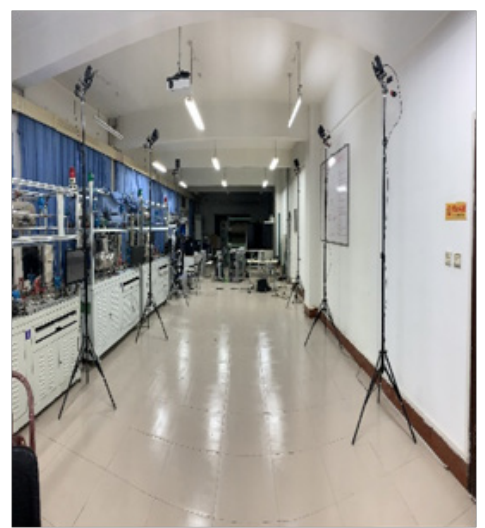

Figure I Human body capture scene.

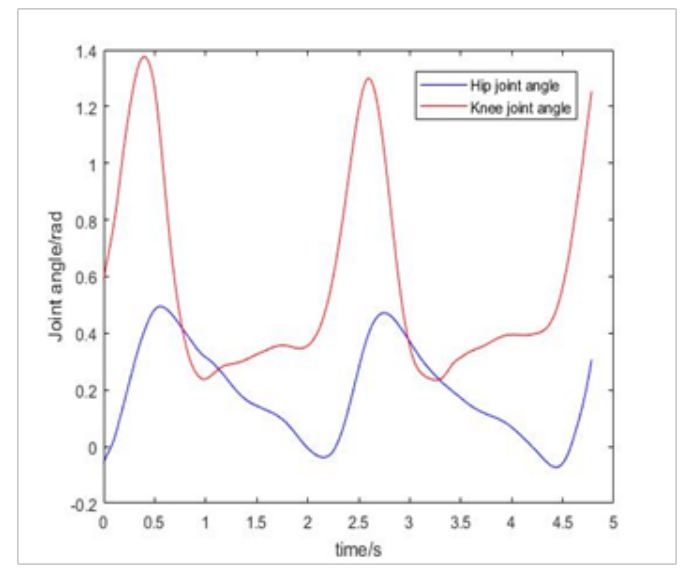

Figure $\mathbf{2}$ The angle of the hip and knee joint.

\section{The lower limb robot dynamics}

This paper considers the low limbs dynamic equations based on the two-link rigid robot model (Figure 3). It can be generally modelled by the following second-order nonlinear differential equation:

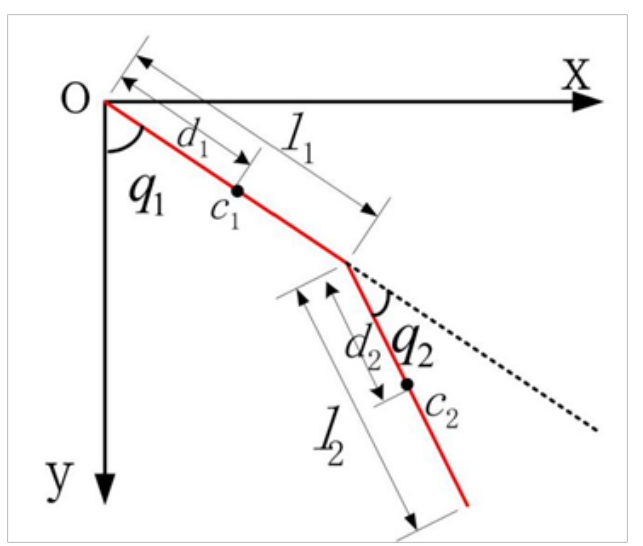

Figure 3 The structure of two-link robot low limb.

$$
D(q) \ddot{q}+C(q, \dot{q}) \dot{q}+G(q)=\tau
$$

where, $q=\left[\begin{array}{ll}q_{1} & q_{2}\end{array}\right]^{T}$, and $q_{1}(t)$ is the joint angle of the hip, $q_{2}(t)$ is the joint angle of the knee. $q=\left[\begin{array}{ll}\dot{q}_{1} & \dot{q}_{2}\end{array}\right]^{T}, \dot{q}_{1}(t)$ is the joint velocity of the hip, $\dot{q}_{2}(t)$ is the joint velocity of the knee. $q=\left[\begin{array}{ll}\ddot{q}_{1} & \ddot{q}_{2}\end{array}\right]^{T}, \ddot{q}_{1}(t)$ is the joint accelerate of the hip, $\ddot{q}_{2}(t)$ is the joint accelerate of the knee. $\tau=\left[\begin{array}{lll}\tau_{c 1}+\tau_{h 1} & \tau_{c 2}+\tau_{h 2}\end{array}\right]^{T}, \quad \tau_{c 1}(t)$ and $\tau_{c 2}(t)$ are the input torque of link knee from the actuator and the human, respectively.

$D(q)$ and $C(q, \dot{q})$ denote the inertial matrix and Coriolis-Centrifugal force vector, respectively. $G(q)$ denote the Gravity matrix.

where,

$$
\begin{gathered}
D(q)=\left[m_{1} d_{1}^{2}+m_{2} l_{1}^{2}+m_{1} d_{2}^{2}+2 m_{1} l_{1} d_{2} \cos q_{2}-\left(m_{2} d_{2}^{2}+m_{1} l_{1} d_{2} \cos q_{2}\right)-\left(m_{2} d_{2}^{2}+m_{1} l_{1} d_{2} \cos q_{2}\right) m_{2} d_{2}^{2}\right] \\
C(q, \dot{q})=\left[\begin{array}{cc}
-2 m_{1} l_{1} d_{2} \dot{q}_{2} \sin q_{2} & m_{1} l_{1} d_{2} \dot{q}_{2} \sin q_{2} \\
m_{1} l_{1} d_{2} \dot{q}_{1} \sin q_{2} & 0
\end{array}\right] \\
G(q)=\left[-\left(m_{1} d_{1}+m_{2} l_{1}\right) g \sin q_{1}-m_{2} d_{2} g \sin \left(q_{1}-q_{2}\right) m_{2} d_{2} g \sin \left(q_{1}-q_{2}\right)\right]
\end{gathered}
$$


where, $m_{1}$ and $m_{2}$ are the quality of robot thigh bar and robot calf rod, $l_{1}$ and $l_{2}$ are the length of robot thigh and robot calf, $d_{1}$ denotes the distance between the joint hip and to the centre of mass of link hip, $d_{2}$ denotes the distance between the joint knee and to the centre of mass of link knee.

According to the dynamic characteristics of the robot system, there is a parameter vector that depends on the lower limb parameters of the robot, so that $D(q), C(q, \dot{q}), G(q)$ satisfy the following linear relationship,

$$
D(q) \vartheta+C(q, \dot{q}) \rho+G(q)=\Phi(q, \dot{q}, \vartheta, \rho) P
$$

where, $\Phi(q, \dot{q}, \vartheta, \rho) \in R^{2 \times 3}$ is the regression matrix of functions of known joint variables, which is the known function matrix of generalized coordinates of the robot and its reciprocal of each order, $P \in R^{3 \times 1}$ is an unknown time-invariant system describing the mass characteristics of the robot.

\section{Lower limb robot control system}

The designed lower limb rehabilitation robot control system is shown in Figure 4. In the control system, including the NOKOV 3D infrared passive optical motion capture system to obtain the desired trajectory of the human, the human comfort function to compensate the interaction force between the human and the robot, the controller is designed for comfort. The sum of the outputs of the controller and human comfort function is fed to the robot leg as the control input signal, namely $\tau=\tau_{c}+\tau_{h}$.

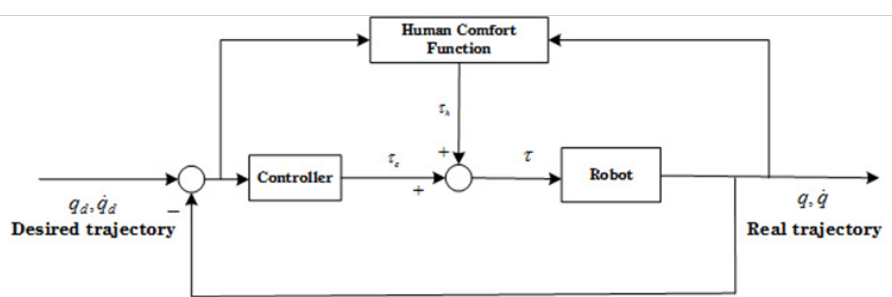

Figure 4 The designed lower limb rehabilitation robot control system.

\section{The comfort function}

The comfort level of the human body wearing rehabilitation training robot greatly determines the rehabilitation efficiency, so this paper proposes a comfort function,

$$
\tau_{h}=a \dot{e}(t)+b e(t)
$$

where, a and b are positive quantities, $\dot{e}=\dot{q}_{d}-\dot{q}, e=q_{d}-q, q_{d}$ and $\dot{q}_{d}$ denote angle and angular velocity of the human trajectory, respectively, $q$ and $\dot{q}$ are the actual angel position and angular velocity, respectively.

\section{Control system design}

According to the second type Lagrange equation, the dynamic model of two link series manipulator can be described by the following equation,

$$
D(q) \ddot{q}+C(q, \dot{q}) \dot{q}+G(q)=\tau_{c}
$$

Introduce filter error $\mathrm{r}(\mathrm{t})$,

$$
r(t)=m \dot{e}(t)+n e(t)
$$

then,

$$
\dot{r}(t)=m \ddot{e}(t)+n \dot{e}(t)
$$

In the formula, $\mathrm{m}, \mathrm{n}$ are positive filter gain. The formula (5) and (6) can also be expressed as

$$
\begin{aligned}
& \dot{q}(t)=\dot{q}_{d}(t)+\frac{1}{m}[n e(t)-r(t)] \\
& \ddot{q}(t)=\ddot{q}_{d}(t)+\frac{1}{m}[n \dot{e}(t)-\dot{r}(t)]
\end{aligned}
$$

Substituting the above two formulas into the control system's dynamic model (4)

$D(q) \ddot{q}+C(q, \dot{q}) \dot{q}+G(q)=D(q)\left\{\ddot{q}_{d}(t)+\frac{1}{m}[n \dot{e}(t)-\dot{r}(t)]\right\}+C(q, \dot{q})\left\{\dot{q}_{d}(t)+\frac{1}{m}[n e(t)-r(t)]\right\}+G(q)=\tau_{c}$

To simplify the formula (9),

$D(q) \frac{1}{m} \dot{r}(t)+C(q, \dot{q}) \frac{1}{m} r(t)+\tau_{c}=D(q)\left[\ddot{q}_{d}(t)+\frac{1}{m} n \dot{e}(t)\right]+C(q, \dot{q})\left[\dot{q}_{d}(t)+\frac{1}{m} n e(t)\right]+G(q)$

From the formula (2), order,

$$
\begin{gathered}
\vartheta=\ddot{q}_{d}+\frac{1}{m} n \dot{e}=\left[\begin{array}{c}
\ddot{q}_{d 1}+\frac{1}{m} n \dot{e}_{1} \\
\ddot{q}_{d 2}+\frac{1}{m} n \dot{e}_{2}
\end{array}\right]=\left[\begin{array}{c}
\vartheta(1) \\
\vartheta(2)
\end{array}\right], \\
\rho=\dot{q}_{d}+\frac{1}{m} n e=\left[\begin{array}{c}
\dot{q}_{d 1}+\frac{1}{m} n e_{1} \\
\dot{q}_{d 2}+\frac{1}{m} n e_{2}
\end{array}\right]=\left[\begin{array}{c}
\rho(1) \\
\rho(2)
\end{array}\right]
\end{gathered}
$$

Substituting the above formula into equation (2),

$D(q)\left(\ddot{q}_{d}+\frac{1}{m} n \dot{e}\right)+C(q, \dot{q})\left(\dot{q}_{d}+\frac{1}{m} n e\right)+G(q)=\Phi(q, \dot{q}, \vartheta, \rho) P$

make $\mu=\frac{g}{l_{1}}$,then

$D(q) \vartheta+C(q, \dot{q}) \rho+G(q)=\left[\begin{array}{lll}\Phi_{11} & \Phi_{12} & \Phi_{13} \\ \Phi_{21} & \Phi_{22} & \Phi_{23}\end{array}\right]\left[\begin{array}{l}P_{1} \\ P_{2} \\ P_{3}\end{array}\right]=\Phi(q, \dot{q}, \vartheta, \rho) P$

where,

$$
\begin{aligned}
& \Phi_{11}=\vartheta(1)+\mu \sin q_{2} \\
& \Phi_{12}=(2 \vartheta(1)-\vartheta(2)) \cos q_{2}-(2 \rho(1)-\rho(2)) \dot{q}_{2} \sin q_{2}-\mu \sin \left(q_{1}-q_{2}\right) \\
& \Phi_{13}=\vartheta(1)-\vartheta(2) \\
& \Phi_{21}=0 \\
& \Phi_{22}=-\vartheta(1) \cos q_{2}-\rho(1) \dot{q}_{1} \sin q_{2}+\mu \sin \left(q_{1}-q_{2}\right) \\
& \Phi_{23}=\vartheta(2)-\vartheta(1)
\end{aligned}
$$

Substituting equation (11) into (10), the open-loop error system equation can be obtained,

$$
D(q) \frac{1}{m} \dot{r}(t)+C(q, \dot{q}) \frac{1}{m} r(t)=\Phi(q, \dot{q}, \vartheta, \rho) P-\tau_{c}
$$

where, $P \in R^{3 \times 1}$ is the minimum inertia parameter of the lower limb robot system, which can be identified by the adaptive law. The following controller is designed for the open-loop error system, 


$$
\tau_{c}=\Phi(q, \dot{q}, \vartheta, \rho) \hat{P}(t)+\beta r(t)
$$

where, $\beta$ is the positive control gain, $\hat{P}(t)$ is the inertia parameter estimation vector of low limb robot, which is also identified by the adaptive law.

Then the closed-loop control system can be obtained

$$
D(q) \frac{1}{m} \dot{r}(t)+C(q, \dot{q}) \frac{1}{m} r(t)=\Phi(q, \dot{q}, \vartheta, \rho) \tilde{P}(t)-\beta r(t)
$$

where, $\tilde{P}(t)=P(t)-\hat{P}(t)$ is the estimation vector of the uncertain inertial parameter. In this case, the parameter estimation rate was taken for

$$
\dot{\hat{P}}(t)=\Gamma \Phi^{T}(q, \dot{q}, \vartheta, \rho) r(t)
$$

where, $\Gamma \in R^{3 \times 3}$ is the positive definite matrix. Take the Lyapunov function of the closed-loop system as

$$
V(t)=\frac{1}{2} r^{T} \frac{1}{m} D(q) r(t)+\frac{1}{2} \tilde{P}^{T} \Gamma^{-1} \tilde{P}
$$

Since $D(q)$ and $\Gamma$ are positive definite, so $V(t) \geq 0$. Then

$$
\dot{V}(t)=r^{T} \frac{1}{m} D(q) \dot{r}+\frac{1}{2} r^{T} \frac{1}{m} \dot{D}(q)+\tilde{P}^{T} \Gamma^{-1} \tilde{P}
$$

The following equation can be expressed from the equation (16)

$$
\dot{\tilde{P}}(t)=\dot{P}(t)-\dot{\hat{P}}(t)=-\dot{\hat{P}}(t)
$$

Substituting the equation (15) and (19) into the equation (18) can be obtained

$$
\begin{gathered}
\dot{V}(t)=\frac{1}{m} r^{T}(-C r+m \Phi \tilde{P}-m \beta r)+\frac{1}{2} r^{T} \frac{1}{m} \dot{D}(q) r+\tilde{P}^{T} \Gamma^{-1}\left(-\Gamma \Phi^{T} r\right) \\
=\frac{1}{m} r^{T}\left[\frac{1}{2} D(q)-C(q, \dot{q})\right] r-\beta r^{T} r+\left[r^{T} \Phi \tilde{P}-\tilde{P}^{T} \Phi^{T} r\right]
\end{gathered}
$$

According to the dynamic characteristics of the Coriolis force matrix, $D(q)-2 C(q, \dot{q})$ is a skew symmetry matrix, so $r^{T}\left(\frac{1}{2} \dot{D}(q)-C(q, \dot{q})\right) \dot{r}=0$. Because $r^{T} \Phi \tilde{P} \in R$, so $r^{T} \Phi \tilde{P}=\tilde{P}^{T} \Phi^{T} r$. Then the equation (20) can be simplified as

$$
\dot{V}(t)=-\beta r^{T} r=-\beta\|r\|^{2} \leq 0
$$

So the closed-loop control system is globally stable. $V(t)$ decays exponentially with time because of $V(t) \geq 0$ and its derivative $\dot{V}(t) \leq 0$. Then $V(t) \in L_{\infty}$ and its derivative $\infty$ - norm is bounded. Therefore $r(t) \in L_{\infty} \cap L_{2}, \dot{r}(t) \in L_{\infty}$ can be obtained from $\tilde{P}(t), \dot{\tilde{P}}(t)$ , $\tau_{c}(t) \in L_{\infty}$. From Barbalat theorem, $\lim _{t \rightarrow \infty} r(t)=0$. According to the properties of the first order linear filter, $e(t)$ and $\dot{e}(t)$ will decay to zero in the same way. So as to achieve the goal of tracking control whenever the initial position of the system.

From equation (29), we can know that $r(t)$ converges exponentially to zero, according to the nature of the filter variable $r(t), e(t)$ and $\dot{e}(t)$ will decay to zero in the same way. So as to achieve the goal of tracking control whenever the initial position of the system.

\section{Simulation-based experimental data}

In this paper, the subject is a healthy woman, whose height is $165 \mathrm{~cm}$ and weight is $52 \mathrm{~kg}$. During the experiment, the subject wore black leggings, whose hands on chest and their feet walking parallel to each other naturally. From section 2, the hip joint $q_{h d}$ and knee joint $q_{k d}$ can be obtained, which as the desired track of the controller, $q_{h d}=a_{0}+a_{1} * \cos (\omega * t)+b_{1} * \sin (\omega * t)+a_{2} * \cos (2 * \omega * t)+b_{2} * \sin (2 * \omega * t)$

$+a_{3} * \cos (3 * \omega * t)+b_{3} * \sin \left(3 * \omega^{*} t\right)+a_{4} * \cos \left(4 * \omega^{*} t\right)+b_{4} * \sin (4 * \omega * t)$

$+a_{5} * \cos \left(5 * \omega^{*} t\right)+b_{5} * \sin \left(5 * \omega^{*} t\right)+a_{6} * \cos \left(6 * \omega^{*} t\right)+b_{6} * \sin \left(6 * \omega^{*} t\right)$

$+a_{7} * \cos (7 * \omega * t)+b_{7} * \sin (7 * \omega * t)+a_{8} * \cos (8 * \omega * t)+b_{8} * \sin (8 * \omega * t)$

\begin{tabular}{|c|c|c|}
\hline$a_{0} / c_{0}$ & $0.2275 / 0.7813^{w / \varpi}$ & $1.51 / 1.071$ \\
\hline$a_{1} / b_{1}$ & $-0.05504 /-0.04065 c_{1} / d_{1}$ & $0.4 \mid 56 /-0.2492$ \\
\hline$a_{2} / b_{2}$ & $0.0504 \mathrm{I} / 0.08886 c_{2} / d_{2}$ & $0.2622 /-0.4546$ \\
\hline$a_{3} / b_{3}$ & $-0.07493 / 0.1694 c_{3} / d_{3}$ & $-0.165 I /-0.01536$ \\
\hline$a_{4} / b_{4}$ & $0.07383 /-0.06247 c_{4} / d_{4}$ & $-0.2246 /-0.06722$ \\
\hline$a_{5} / b_{5}$ & $-0.09099 / 0.1132 c_{5} / d_{5}$ & $-0.1654 / 0.0392$ \\
\hline
\end{tabular}

$q_{k d}=c_{0}+c_{1} * \cos (\varpi * t)+d_{1} * \sin (\varpi * t)+c_{2} * \cos (2 * \varpi * t)+d_{2} * \sin (2 * \varpi * t)$

$+c_{3} * \cos (3 * \varpi * t)+d_{3} * \sin (3 * \varpi * t)+c_{4} * \cos (4 * \varpi * t)+d_{4} * \sin (4 * \varpi * t)$

$+c_{3} * \cos (3 * \varpi * t)+d_{3} * \sin (3 * \varpi * t)+c_{4} * \cos (4 * \varpi * t)+d_{4} * \sin (4 * \varpi * t)$

$+c_{5} * \cos (5 * \varpi * t)+d_{5} * \sin (5 * \varpi * t)+c_{6} * \cos (6 * \varpi * t)+d_{6} * \sin (6 * \varpi * t)$

$+c_{7} * \cos (7 * \varpi * t)+d_{7} * \sin (7 * \varpi * t)+c_{8} * \cos (8 * \varpi * t)+d_{8} * \sin (8 * \varpi * t)$

The specific parameters of the $q_{h d}$ and $q_{k d}$ are shown in Table 1,

Table I The parameters of joint angle desired trajectory

parameter numerical parameter numerical

The structural parameters of the lower limb rehabilitation training robot are: $m_{1}=4 \mathrm{~kg}, l_{1}=0.5 \mathrm{~m}, d_{1}=0.25 \mathrm{~m}, m_{2}=3.1 \mathrm{~kg}, l_{2}=0.4 \mathrm{~m}$ ,$d_{1}=0.2 \mathrm{~m}$. And the gravitational acceleration is $9.8 \mathrm{~m} / \mathrm{s}$. Thus $p_{1}=1.149, p_{2}=0.124, p_{3}=0.31, p_{4}=-24.99, p_{5}=6.076$. The comfort function coefficient is: $a=0.8$ and $b=0.1$. The linear filter gain is: $m=200$ and $n=25$. The gain of the control law is: $\beta=40$. And the gain update matrix take $\Gamma=\operatorname{diag}\left[\begin{array}{lll}3 & 3 & 3\end{array}\right]$. Initial states of the hip and knee joints of the lower limb rehabilitation training robot were taken $\left(q_{h d}, q_{k d}\right)=(0,0)$ and $\left(\dot{q}_{h d}, \dot{q}_{k d}\right)=(0,0)$. The simulation results are shown in the Figures 5-10.

As can be seen from the simulation results, the trajectory of the hip joint and the knee joint quickly tracks to the desired trajectory while the inertial parameters keep changing, and the tracking trajectory error quickly converges to zero. The velocity of the joint trajectory can also track the velocity change of the desired trajectory rapidly and the control input is relatively stable. Thus, the effectiveness of the control method designed in this paper is verified, and the prototype worn by the subject is comfortable, so as to verify the scientificity of the research based on comfort function in this paper. 

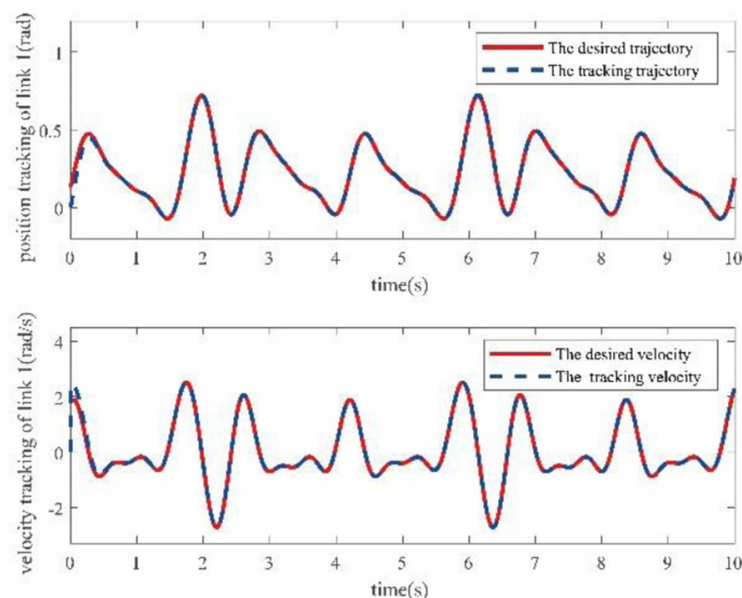

Figure $\mathbf{5}$ The position tracing and velocity tracking of link I.
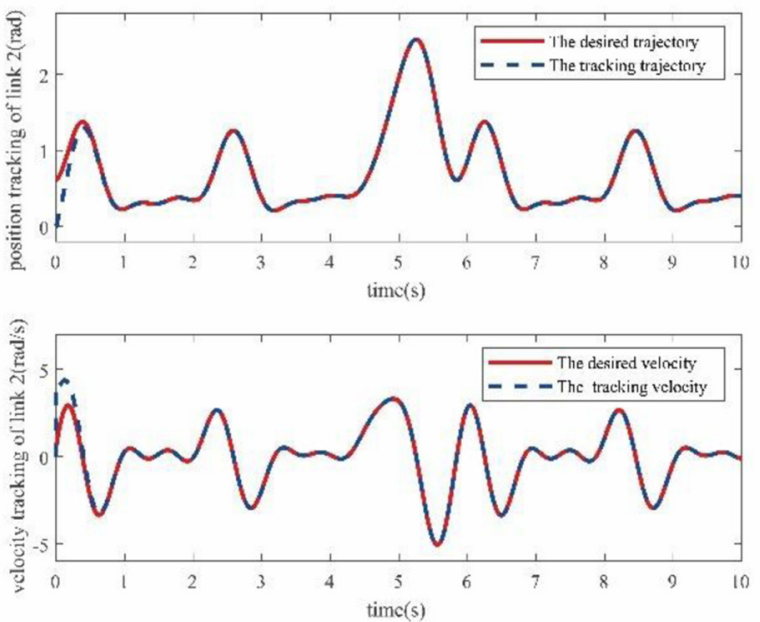

Figure 6 The position tracing and velocity tracking of link 2.

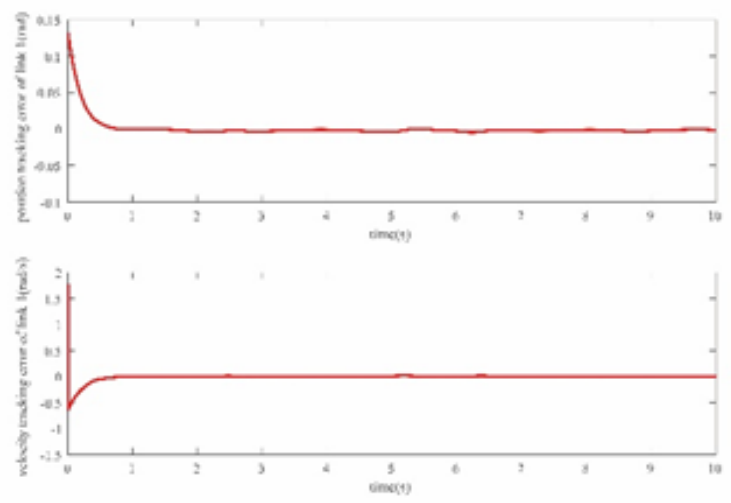

Figure 7 The position tracing error and velocity tracking error of link I.
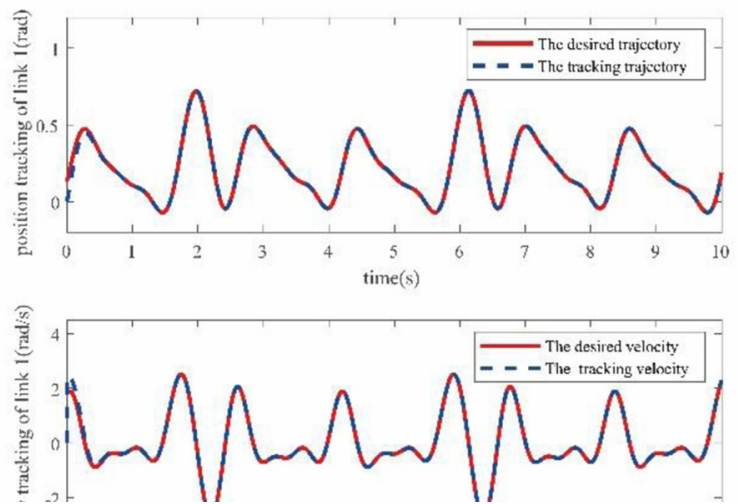

Figure $\mathbf{8}$ The position tracking error and velocity tracking error of link.
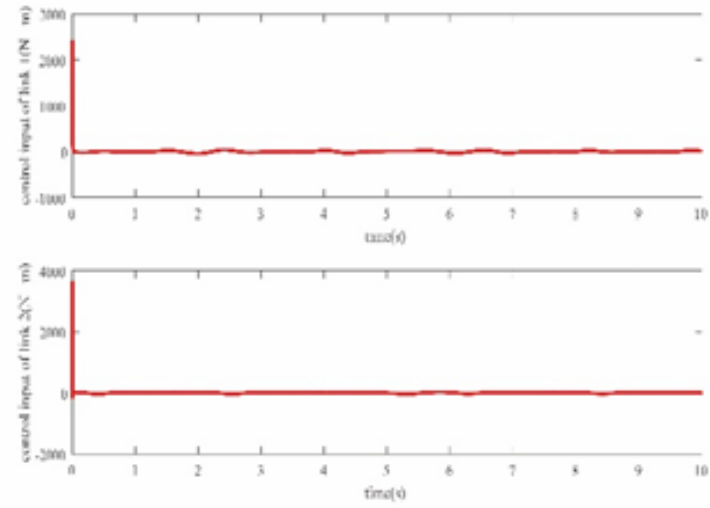

Figure 9 The input torque of link I and link 2
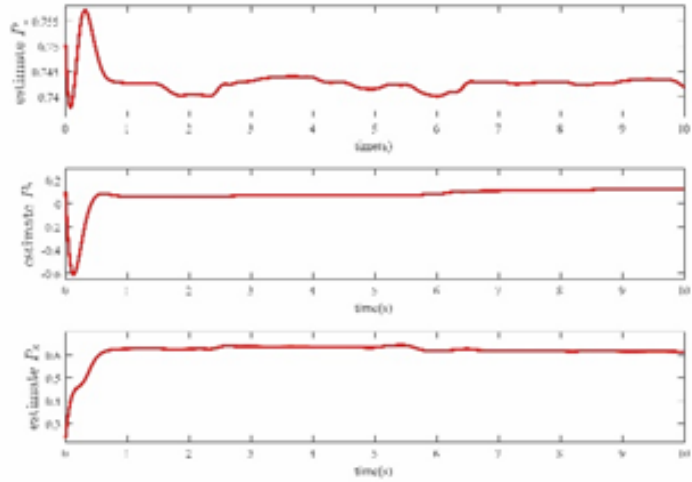

Figure 10 Inertia parameter estimated.

\section{Conclusion}

In this paper, an adaptive control study is designed based on the comfort level of the wearable lower limb rehabilitation robot in the case of uncertain inertial parameters. In the case of minimum inertial parameters, the controller is designed with comfort level as the objective function to ensure the safety and comfort of patients for 
rehabilitation training. Through the simulation experiment, the results show that the controller can track the desired trajectory very well, and there is no speed mutation in the tracking process. The control precision is high, and the subject is comfortable to wear, which verifies the feasibility and effectiveness of the method proposed.

\section{Funding}

The authors would like to thank the Young Backbone Teacher Training Program of Henan Province's Higher Education (2017GGJS117), and National Natural Science Foundation (U1813201) for their support of this work.

\section{Acknowledgments}

None.

\section{Conflicts of interest}

The author declares there are no conflicts of interest.

\section{References}

1. Chen X. Data from the second national sample survey of disabled persons CJH. 2008;19(68):1-3.

2. Xie $\mathrm{C}, \mathrm{Xu} \mathrm{G}$, Liu X. Research progress of early rehabilitation after stroke. Rehabilitation theory and practice in China. 2009;15(10):908-912.

3. Woldag H, Hummelsheim H. Evidence--based physiotherapeutic concepts for improving arm and hand function in stroke patients: a review. J Neurol. 2002;249(5):518-528.

4. Li F. The research of lower limb rehabilitation training robot active control. Shanghai university. 2014;4-15.
5. Meng W, Liu Q, Zhou Z, et al. Recent development of mechanisms and control strategies for robot-assisted lower limb rehabilitation. Mechatronics. 2015;31:132-145.

6. Yan T, Cempini M, Oddo CM, et al. Review of assistive strategies in powered lower-limb orthoses and exoskeletons. Robotics and Autonomous Systems. 2015;64:120-136.

7. Kim CS, Mo EJ, Han SM, et a1. Robust visual servo control of robot manipulators with uncertain dynamics and camera parameters. International Journal of Control, Automation and Systems. 2010;8(2):308313.

8. Urrea C, José P. Design, simulation, comparison and evaluation of parameter identification methods for an industrial robot. Computer \& Electrical Engineering. 2018;67:791-806.

9. Yang C, Ganesh G, Haddadin S, et al. Human-Like Adaptation of Force and Impedance in Stable and Unstable Interactions. Ieee Transactions On Robotics. 2011;27(5):918-930.

10. De Luca A, Siciliano B, Zollo L. PD control with on-line gravity compensation for robots with elastic joints: Theory and experiments. Automatica. 2005;41(10):1809-1819.

11. Zollo L, Siciliano B, De Luca A, et al. PD Control with On-line Gravity Compensation for Robots with Flexible Links. European Control Conference (ECC). 2007;4365-4370.

12. Tang A, Cao Q. Motion control of walking assistant robot based on comfort. Industrial Robot. 2012;39(6):546-579.

13. Yang F, Yuan X G. Human motion control based on maximum comfort. JCADP. 2015;17(2):267-272.

14. Liu Y, Zhang XD. Analysis and research on comfort of rehabilitation robot flexible joint. MRA. 2015;4:9-11. 